\title{
DEM simulation of dense granular flows in a vane shear cell: Kinematics and rheological laws
}

\author{
Fenglei Qi ${ }^{\mathrm{a}, *}$, Sébastien Kiesgen de Richter ${ }^{\mathrm{b}}$, Mathieu Jenny ${ }^{\mathrm{b}}$, Bernhard Peters ${ }^{\mathrm{a}}$ \\ ${ }^{a}$ Faculty of Science, Technology and Communication, University of Luxembourg,2 avenue de l'Université, \\ 4365 Esch-sur-Alzette, Luxembourg \\ ${ }^{b}$ Université de Lorraine, LEMTA, CNRS UMR 7563, Vandoeuvre-lès-Nancy, F-54500, France
}

\begin{abstract}
The rheology of dense granular flows is investigated through discrete element method (DEM) simulation of a vane shear cell. From the simulation, profiles of shear stress, shear rate, and velocity are obtained, which demonstrates that the flow features in the vane shear cell are equivalent to those in the classic annular Couette cell. A novel correlation for the shear viscosity is formulated and leads to a new expression for $\mu^{K T}$ in the kinetic theory analysis. The $\mu^{K T}$ formulation is able to qualitatively capture the $\mu-I$ relation in the shear cell. A correlation length is added in the energy dissipation term to account for the effects of the particle motion correlation. A simplified correlation length model is derived based on DEM results and is compared with the literature. The modified granular kinetic energy equation is able to correctly predict the granular temperature profiles in the shear cell.
\end{abstract}

Keywords:

Annular Couette cell; Discrete element method (DEM); Extended kinetic theory; Granular flow; Rheology

\section{Introduction}

Dense granular flows are widely found in industrial processing equipment such as silos [1, 2], pneumatic conveyors [3], vibrating inclined planes [4] and rotating drums [5]. Difficulties

\footnotetext{
*I am corresponding author

Email addresses: fenglei.qi@uni.lu (Fenglei Qi), sebastien.kiesgen@univ-lorraine.fr (Sébastien Kiesgen de Richter) 
are reported in the simulation of granular flows in such devices due to the lack of a universal rheological model [2, 6]. Rheological behaviors of dry granular flows are often described in three flow regimes, which are distinguished as quasi-static $(I \lesssim 0.001)$, intermediate $(0.001 \lesssim I \lesssim 1.0)$ and collisional $(I \gtrsim 1.0)$ regimes based on an inertial number parameter $I=\dot{\gamma} d_{p} / \sqrt{P / \rho_{p}}$ with $\dot{\gamma}$ being the shear rate, $d_{p}$ particle diameter, $\rho_{p}$ particle intrinsic density, and $P$ the confining pressure [7, 8, 9]. In the quasi-static regime, the particles move slowly and pack densely especially with the action of gravity, and an enduring contact network is often observed. In contrast, the collisional regime is featured by instantaneous and binary particle collisions, and macroscopically the grains behave like a gas. The solid fraction is also reduced as a result of increased particle fluctuation energy. The intermediate regime represents a transition of the particle dynamics and the resultant macroscopic behaviors between quasi-static and collisional regimes. The particle motion in this regime is typically correlated and the particles often have multiply long-lasting contacts in one collision instance [10, 11]. Strongly sheared flows also features anisotropy in the force network, which results in anisotropy of the fabric of contacts [12] and the diffusion of fluctuation energy [13]. Macroscopic understanding of the particle dynamics in this regime is far from satisfactory, making it very challenging to formulate a generalized constitutive model for continuum modeling of the intermediate flow regime [14, 15].

Considering that the intermediate regime, as its name suggests, lies in between the quasistatic regime and the collisional regime, it seems reasonable that the formulated rheology model for the intermediate regime combines the features of the two regimes, and converges to the results for the two regimes at lower and upper limits. The quasi-static regime exhibits a ratio of shear stress to normal stress that is independent of shear rates [16, 17]. The shear stress is computable with the Mohr-Coulumb yield criterion. In the collisional regime, the shear stress depends on inertial properties (such as particle fluctuation energy and strain rate) and can be well predicted by the kinetic theory of granular gases. The correct formation of the rheological model for the intermediate regime is essential to describing the granular flows in all regimes. G.D.R. MiDi [7] suggested a $\mu(I)$ relation, which has successfully described the rheological behaviors of dense granular flows [7, 8, 18]. Its modified versions 
have been applied to modeling of dense granular flows in several flow configurations [1, 2, 3, 19, 20, 21]. The formulation developed by Jop et al. [19] is written as

$$
\mu(I)=\mu_{s}+\frac{\mu_{g}-\mu_{s}}{I_{0} / I+1}
$$

35 It is demonstrated that the new $\mu^{K T}$ formulation is able to satisfactorily recover the $\mu-I$ relation in the presence of shear gradient. 
The correlated motion of adjacent particles in dense granular flows was well reported in length [35, 36, 39], to our knowledge, there is limited research in the literature investigating 
whether the kinetic theory is able to predict nonlocal effects inherent in dense granular flows sheared in an annular shear cell. It is the aim of this research to examine if rheological scaling laws based on the granular temperature can be generated through DEM simulation of a vane shear cell and whether the modifications of the kinetic theory is capable of predicting the nonlocaity.

Understanding various macroscopic behaviors of granular flows benefits from analysis of the underlying particle dynamics. In this contribution, we carry out simulations of granular flows in a vane shear cell using DEM approach. From the DEM simulation, local information such as stresses, strains and granular temperature is extracted from particle dynamics simulations and then is used for rheological model development based on the kinetic theory. In the following, we first describe the simulated system and the DEM model parameters. Next, we present the profiles of the shear stress, shear rate, velocity and effective friction coefficient in the shear cell, which demonstrates the flow similarities between the vane shear cell and the classic annular Couette cell. We then present the development and assessment of the shear viscosity correlation, the energy dissipation rate and the diffusion term based on DEM simulation results.

\section{Methodology}

\subsection{Vane shear cell}

Three-dimensional (3D) DEM simulations of dense granular flows in a vane shear cell are carried out in this research and the results are used to derive rheological models. The simulated system includes a rotating vane and an outer stationary wall, which is shown in Fig. 1. The prototypical apparatus of the modeled shear cell has been used to experimentally study the rheology of granular and suspension systems [40, 41. In this configuration, shear stress is applied to the dry and frictional granular material in the annular gap through the same material that is trapped between the blades. In the modeled shear cell, all dimensions are scaled by particle mean size $\left\langle d_{p}\right\rangle$. The radius of the blade tip $\left(R_{i}\right)$ is $27.5\left\langle d_{p}\right\rangle$ and the outer wall $\left(R_{o}\right)$ is $57.5\left\langle d_{p}\right\rangle$, generating an annular cell of $30\left\langle d_{p}\right\rangle$ width. The vane moves 
at a prescribed rotation speed $\Omega$, while the outer wall remains stationary. In all simulations, the particle mean size $\left\langle d_{p}\right\rangle$ is $1.0 \mathrm{~mm}$ and a polydispersity of $\pm 20 \%$ is adopted to prevent crystallization [42]. The material of all entities including particles and geometrical structures adopts glass material with a density of $2400 \mathrm{~kg} / \mathrm{m}^{3}$. A packing bed is prepared before starting DEM simulations. The procedure is as following: a certain height of dense granular packing is initiated subjecting to gravity at a very slow rotation speed and the bed height $(H)$ is truncated to $38\left\langle d_{p}\right\rangle$ when the flow reaches steady. The design of the system is aimed to numerically replicate flow characteristics of three dimensional granular flows in the annular Couette cell and reduce the system size for affordable simulation time. With the current settings, around 432,000 particles are adopted to construct the granular system. The simulations were initiated with the same packing bed generated at the beginning and the top surface of the granular bed is free to move in all simulations. Pressure is gradually built up due to the gravity in the vertical direction and serves as confining pressure for packing particles. In this contribution, all the variable values such as stresses and solid fractions were extracted above $H_{\min }=10 d_{p}$, which avoids the bottom wall effects on the analysis.

[ Fig. 1 insert here. ]

\subsection{Parameters in numerical experiments by DEM}

The adopted DEM simulation method allows for resolving long-lasting particle collision instances with a well verified spring-dashpot contact model. The Hertz-Mindlin contact model was used in all simulations. Detailed description of the contact model refers to our previous research [43]. The simulation of particle dynamics is affected by parameters in the contact model including Young's modulus $Y$, restitution coefficient $e$, Poisson ratio $\nu$ and sliding friction coefficient $\mu_{p}$. Macroscopically, the granular flow in the shear cell is entirely describable by parameters characterizing material contact properties and the shear state imposed by the vane.

In the Hertz-Mindlin contact model, the normal spring stiffness $k_{n}$ and the tangential stiffness $k_{t}$ are dynamically determined from the Young's modulus, Poisson ratio and collision 
deformation. However, the ratio of the tangential stiffness to the normal stiffness remains a constant, written as $k_{t} / k_{n}=3(1-\nu) /(2-\nu)$, after the Poisson ratio is prescribed. Since the collision deformation is a result of the contact dynamics, the Young's modulus parameter is the only independent variable determining the contact stiffness. To characterize the rigidity of the packing bed, a stiffness number is defined as $\kappa=C\left(P_{m} / Y\right)^{1 / 3}$ with $\mathrm{C}$ equal to 1.69 for the glass material and $P_{m}$ being a characteristic pressure. The detailed derivation of the stiffness number is shown in Appendix B. Previous research suggests $\kappa<0.01$ in order to eliminate influences of the material rigidity on simulation results. With the characteristic pressure defined as $P_{m}=0.6 \rho_{p} g H_{m}$ and $H_{m}=25\left\langle d_{p}\right\rangle$, the influences of the material rigidity on simulation results are found insignificant when the Young's modulus is set to be $2 \times 10^{7}$ and the stiffness number is equal to 0.044 as discussed in Appendix B. The Poisson ratio of the glass material is a constant and the value $\nu=0.22$ is taken from a previous DEM research [44]. Regarding the restitution coefficient, it has been reported to have little impact on simulation results 16 and a constant value of 0.8 is used in all simulations. A constant sliding friction coefficient is used with the value of 0.2 and the simulation results match with previous researches as discussed in later section. Moreover, any long range interaction between two particles such as the capillary interaction is not considered in this study.

The prescribed shear rate from the vane rotation is measured by a dimensionless shear velocity defined as:

$$
V_{\theta}=\Omega\left\langle d_{p}\right\rangle \sqrt{1 /\left(g H_{m}\right)}
$$

with $g$ being the gravitational acceleration. $V_{\theta}$ is varied from 0.0005 to 0.4 in DEM simulations. As a result, the inertial number at the first layer adjacent to the inner boundary varies in a range of $[0.003,0.25]$ and the solid fraction lies in the range of $[0.55,0.64]$, which covers the intermediate regime.

The flow patterns in the shear cell were visually checked within the range of the dimensionless velocity studied here, which can be seen in Appendix A. It is worth noticing that the dilation of the granular bed is insignificant and the free surface remains flat, which suggests 
that the granular flow in the studied range is not influenced by vortex flow features observed in other research [45].

\subsection{Derivation of flow properties}

Flow properties are carefully derived from samples collected in DEM simulations, which contains collision properties of each interactive pair of particles and dynamic properties of each particle. Starting from the initial packing bed and a prescribed rotational speed of the vane, the granular flow in the shear cell gradually approaches a steady state at which the time-volume averaged flow properties do not vary in time. The justification of the steady state is performed by monitoring the time evolution of variables such as stresses, solid fraction and granular temperature. The sampling only starts when the granular flow is shown to reach steady state.

Analysis of the granular flow in the annular Couette cell suggests that the averaged flow properties have no variations in the azimuthal direction due to the axial symmetry of the system as shown in Fig. 1. The samples are therefore collected in a time series of $N$ and at location $(r, z)$ within a spatial interval of $\Delta r=2\left\langle d_{p}\right\rangle$ and $\Delta z=2\left\langle d_{p}\right\rangle$. The selected spatial interval size is identical to the averaging domain size adopted in previous studies [16, 12, which satisfies the local homogeneity requirement for deriving kinematic properties [46]. In addition, an independence verification of the selected interval size is also provided in Appendix C. An averaged flow property is achieved by doing the time-volume average of the collected $N$ samples at the prescribed interval centrally located at $(r, z)$, which is generally written as

$$
\langle f(r, z)\rangle_{t, V}=\frac{1}{N V} \sum_{i=1}^{N} f(r, z),
$$

where $V$ is the interval volume centrally located at $(r, z)$. For example, with $f(r, z)$ being the total volume occupied by particles at a time, the time-volume average of the property generates the averaged solid fraction. If the flow property $f$ is a particle-based variable such 
as particle velocity, the time average operator is written as

$$
\langle f(r, z)\rangle_{t}=\frac{1}{\sum_{i=1}^{N} \sum_{j=1}^{M_{i}} V_{j}} \sum_{i=1}^{N} \sum_{j=1}^{M_{i}} V_{j} f(r, z),
$$

where $M_{i}$ is the number of particles collected at a sampling time $i$ and $V_{j}$ the volume of a particle positioning within the interval. The time-volume average operator of particle-based variables is formulated as

$$
\langle f(r, z)\rangle_{t, V}=\frac{1}{N V} \sum_{i=1}^{N} \sum_{j=1}^{M_{i}} f(r, z) .
$$

The time-volume average is adopted when calculating variables such as stresses due to particle fluctuation velocity. Similarly, for interaction-based variables such as stresses due to particle long-lasting contacts, the time-volume average operator is achieved by replacing $M_{i}$ with $X_{i}$ in Eq. (5), where $X_{i}$ is the number of particle interactions contained in the interval volume at a sampling time $i$. For convenience, the time average operator and the time-volume average operator are not distinguished from each other. Moreover, the average bracket is left out in the rest of the paper. Wherever a misunderstanding potentially arises due to this writing, an explanation of the variable is provided for clarification.

The total stress tensor is calculated as

$$
\boldsymbol{\sigma}=\frac{1}{N V} \sum_{i=1}^{N}\left[\sum_{j=1}^{M_{i}} m \boldsymbol{v}_{j}^{\prime} \otimes \boldsymbol{v}_{j}^{\prime}-\sum_{x=1}^{X_{i}} \boldsymbol{F}_{x} \otimes \boldsymbol{l}_{x}\right],
$$

where, $m$ is particle mass, $\boldsymbol{v}_{i}^{\prime}$ instantaneous particle velocity fluctuation, $\boldsymbol{F}_{x}$ the contact force in a collision, and $\boldsymbol{l}_{x}$ the branch vector connecting the centers of particles in the collision pair. The first and second terms represent the dynamic and static contributions, respectively. The stress $\boldsymbol{\sigma}$ is decomposed into an isotropic part $P$ and a deviatoric part $\boldsymbol{\tau}$ written as:

$$
P=-\frac{1}{3} \operatorname{tr} \boldsymbol{\sigma}, \boldsymbol{\tau}=\boldsymbol{\sigma}+P \boldsymbol{I}
$$


The strain tensor is calculated as

$$
\boldsymbol{S}=\frac{1}{2 \sum_{i=1}^{N} X_{i}} \sum_{i=1}^{N} \sum_{j=1}^{X_{i}}\left(\nabla \boldsymbol{v}_{j}+\nabla^{T} \boldsymbol{v}_{j}\right)
$$

where, $\nabla \boldsymbol{v}_{j}$ is a velocity gradient calculated from the velocities and positions of a pair of particles, in which one of the particles is particle $j$ and the other one is selected from its neighbor particles. $X_{i}$ represents the total number of particle pairs obtained in a sampling from DEM simulations. The selection of a pair of particles for calculating velocity gradient is different from the interactive particle pairs used for calculating static stress tensor. When two particles that are close to each other or at collision, a strong correlated motion is observed. In order to eliminate the correlation effect on the calculation of the velocity gradient, two particles in the interval volume that are at least one particle diameter away from each other in each direction are considered as a valid pair. Letting $c_{i},(i=r, \theta, z)$ being the coordinates of a particle center, the selection criterion is formulated as $d_{p}<$ $\left|c_{1 i}-c_{2 i}\right|<2 d_{p}$ for particle 1 and 2 to be a valid pair.

The consistency of the local averaged quantities is guaranteed by collecting samples over a characteristic duration $\Delta t$, which is determined in such a way that the local deformation meets the condition: $\dot{\gamma} \Delta t>10 d_{p}$. Due to the shearing localization, the deformation is different at various locations in $r$. Only the regions within which the consistency condition is satisfied are analyzed and are identified as reaching 'steady state' [16]. A number of 100 samples striding the same time interval is collected in each numerical experiment for deriving macroscopic flow properties.

\section{Flow properties in vane shear cell}

The granular flow in the shear cell is characterized by flow properties such as solid fraction, velocity, stress and strain fields. Referring to Eq. (7), the equivalent shear stress is defined as $\tau=\|\boldsymbol{\tau}\|=\sqrt{0.5 \sum_{i} \sum_{j} \tau_{i j} \tau_{i j}}$ and similarly the equivalent shear rate is written as $\dot{\gamma}=2\|\boldsymbol{D}\|=\sqrt{2 \sum_{i} \sum_{j} D_{i j} D_{i j}}$, where tensor $\boldsymbol{D}$ is the deviatoric part of strain tensor $\boldsymbol{S}$. 
Typical flow features of an annular Couette cell is recovered in the DEM simulation of the vane shear cell. In Fig. 2, the differences between the stress component $\tau_{r \theta}$ and the equivalent shear stress are insignificant, which implies that the other deviatoric stress components are trivial. A similar observation is found in the comparison of the strain component $\left|D_{r \theta}\right|$ and the equivalent shear rate. Therefore, the deviatoric stress tensor and the deviatoric strain tensor is well algined with each other. In the shear zone, the principal motion of particles is in the azimuthal direction and the motion in both radial and vertical direction is negligible. The continuum governing equations of granular flows in the classic annular Couette shear cell is obtained after ignoring trivial terms:

$$
\begin{aligned}
& -\rho_{p} \phi \frac{v_{\theta}^{2}}{r}=\frac{\partial \sigma_{r r}}{\partial r}+\frac{\sigma_{r r}-\sigma_{\theta \theta}}{r} \\
& \tau_{r \theta}=\tau_{r=R_{i}}\left(\frac{R_{i}}{r}\right)^{2}
\end{aligned}
$$

where $\rho_{p}, \phi$ are intrinsic density of particles and solid fraction, separately.

Fig. 3 illustrates the variation of the azimuthal velocity $v_{\theta}$ with the radius in the shear cell. The fitted velocity profiles imply that the azimuthal velocity decays following a Gaussian function instead of an exponential function, which is clearly shown in the semi-log figure. The predicted velocity profiles are very similar to velocity profiles obtained from DEM simulations of a two-dimensional (2D) annular Couette cell [16] and experimental measurements in a 2D annular shear cell apparatus [23. The similarities in the velocity profiles suggest that the flow features in the vane shear cell are analogical to those observed in annular Couette shear cells. Although it is not directly shown from the fitted equation, a partial slip boundary is indicated at $r=R_{i}$ with a slippery coefficient varying from 0.62 to 0.706. Realizing that the gradient of the velocity occurs in $r$ direction, the shear rate $\dot{\gamma}$ is estimated from $\dot{\gamma}(r)=-r \frac{\partial}{\partial r}\left(v_{\theta}(r) / r\right)$ by utilizing the fitted equations for azimuthal velocity 
$v_{\theta}(r)$, and is compared with the equivalent shear rate extracted by Eq. (8) in Fig. 2. A satisfactory agreement is observed from the comparison, validating the approach adopted for obtaining macro-scale flow properties from DEM simulations.

[ Fig. 3 insert here. ]

The shear stress is inversely related to $r^{2}$ according to Eq. 10). This relation is recovered in the fitted equations for the equivalent shear stress as shown in Fig. 2, which again validates the Couette analogy of the vane shear cell. In Fig. 4, the normal pressure $\sigma_{r r}$ has

little variance in $r$ direction. After letting $\partial \sigma_{r r} / \partial r=0$, Eq. (9) predicts $\sigma_{\theta \theta}=\sigma_{r r}+\rho_{p} \phi v_{\theta}^{2}$, in which the second term accounts for the dynamic pressure contribution. In the shear zone, comparison of normal stresses in Fig. 4 implies that $\sigma_{\theta \theta}>\sigma_{r r}>\sigma_{z z}$.

[ Fig. 4 insert here. ]

\section{Failure of the $\mu(I)$ law in the shear cell}

The isotropic pressure $P$, the equivalent shear stress $\tau$ and the equivalent shear rate $\dot{\gamma}$ are gathered from the simulation data and used to calculate the effective friction coefficient $\mu$ and the inertial number $I$. Fig. 5 shows the relationship between the two variables and compares the predicted results with both experimental measurements and simulations from previous research [47, 7, 48, 49]. A good agreement is observed between the predicted relation at the inner wall $\mu_{w}\left(I_{w}\right)$ and the literature results. With these data, the parameters in Eq. (1) are estimated: $\mu_{s}=\tan \left(20.1^{\circ}\right), \mu_{g}-\mu_{s}=0.4438$ and $I_{o}=0.55$.

\section{[ Fig. 5 insert here. ]}

The shear zone in the vane shear cell is demonstrated in Fig. 1 and characterized by the velocity profiles in Fig. 3 to be around 8-12 particle size width. In the shear zone 
away from the inner wall, the relationship between the effective friction coefficient and the inertial number is well predicted by Eq. (1) with the estimated parameters when the inertial number is above 0.1 approximately. However, the prediction of $\mu(I)$ relation deviates from the DEM results when the inertial number is below 0.1 in the shear zone. Two sub-regions are identified similarly to Koval's study [16]: inertial region with $\mu>\mu_{s}$ and quasi-static region with $\mu<\mu_{s}$. Clearly, the $\mu(I)$ law is not able to capture the underlying physics in the transition from the inertial region to the quasi-static region. The observed particle motions in the quasi-static zone are incurred by the correlated motion of particles, which are perceived as the nonlocal effects in previous research [16, 17, 50. It is also confirmed that the relation between the effective friction coefficient and the inertial number is not monotonic in the shear cell. Therefore, the viscosity written as $P \mu(I) / \dot{\gamma}$ cannot be correctly predicted from the $\mu(I)$ law.

\section{Scaling laws revealed by DEM simulations}

Granular temperature $T$ is defined as

$$
T=\frac{1}{3}\left(\delta V_{r}^{2}+\delta V_{\theta}^{2}+\delta V_{z}^{2}\right)
$$

where, $\delta V_{r}, \delta V_{\theta}$, and $\delta V_{z}$ represent velocity fluctuations in radial, azimuthal and vertical directions. In this section, scaling relations involving the granular temperature and solid fraction such as $T-\dot{\gamma}$ and $\eta-T$ are obtained from DEM simulations.

\section{1. $T-\dot{\gamma}$ relation}

For simple granular shear flows, the modified kinetic theory predicts that $T /\left(\dot{\gamma} d_{p}\right)^{2}$ is a function of solid fraction [15]. In the vane shear cell which resembles an annular Couette cell, the relation of $T /\left(\dot{\gamma} d_{p}\right)^{2}$ and $\phi_{c}-\phi$ is shown in Fig. 6, where $\phi_{c}$ represents a critical solid fraction and is found to be equal to 0.64 in this research. It is derived from DEM data that $T /\left(\dot{\gamma} d_{p}\right)^{2}=c\left(\phi_{c}-\phi\right)^{-3.2}$ when the solid fraction $\phi>0.59$. The granular temperature 
$T$ and the shear rate $\dot{\gamma}$ have the following relation when the solid fraction $\phi>0.59$ :

$$
T=M\left(\phi, \mu_{p}, e\right)\left(\dot{\gamma} d_{p}\right)^{2}
$$

where, $M\left(\phi, \mu_{p}, e\right)$ is written to account for influences of the friction coefficient $\mu_{p}$ and the restitution coefficient $e$ of the granular material, which are currently not quantified. The power dependence of $T /\left(\dot{\gamma} d_{p}\right)^{2}$ on $\phi_{c}-\phi$ is much smaller than the power of -0.5 reported by Chialvo and Sundaresan [15] in the DEM simulation of simple granular shear flows. When $\phi<0.59$, the dependence on $\phi_{c}-\phi$ starts to disappear, suggesting that $T \sim \dot{\gamma}^{2}$ and is in consistent with kinetic theory predictions.

\subsection{Shear viscosity}

The shear viscosity is defined as

$$
\eta=\frac{\tau}{\dot{\gamma}}=\frac{\|\boldsymbol{\tau}\|}{2\|\boldsymbol{D}\|}
$$

where, $\tau$ and $\dot{\gamma}$ are equivalent shear stress and equivalent shear rate, separately. Kinetic theory analysis of dense granular flow [51] shows that the viscosity has $\eta \sim \eta_{o} P /\left(n_{c} d_{p}^{2} T^{1 / 2}\right)$ with $n_{c}$ being number density at the random close packing configuration. The dimensionless coefficient $\eta_{o}$ diverges when the packing approaches the close random packing and it is taken into account with the formulation $\eta_{o} \sim\left(1-n / n_{c}\right)^{-b} \sim\left(1-\phi / \phi_{c}\right)^{-b}$. Dimensional analysis implies $\eta \sqrt{T} /\left(P d_{p}\right) \sim\left(1-\phi / \phi_{c}\right)^{-b}$.

The scaled viscosity $\eta \sqrt{T} /\left(P d_{p}\right)$ is plotted against the solid fraction variable $1-\phi / \phi_{c}$ in Fig. 7 and is compared with the relation obtained from the kinetic theory analysis. The result indicates that the relation satisfactorily predicts the observations from the DEM simulation at dense packing regions, which are not far from random close packing $\left(\phi \sim \phi_{c}\right)$. However, the DEM data saturates when the solid friction decreases to $0.9 \phi_{c}$ approximately and deviates from the kinetic theory analysis of dense granular flow. The region where the data saturates implies a transition from the intermediate regime to the collisional regime. In the collisonal regime, the kinetic theory of rapid granular flows derives $\eta=\rho_{p} d_{p} \phi \sqrt{T / \pi} f(\phi, e)$, 
where the function of the solid fraction has various formulations. One formulation reported by Gidaspow [52] is written as:

$$
f(\phi, e)=1.016 \frac{5 \pi}{96} \frac{\left(1+4 / 5(1+e) \phi g_{0}\right)\left(1+8 / 5 \phi g_{0}\right)}{\phi^{2} g_{0}}+\frac{4}{5} g_{o}(1+e),
$$

$$
\eta=\eta_{1}+\eta_{2}=\frac{a P d_{p}}{\sqrt{T}}\left(1-\phi / \phi_{c}\right)^{-b}+\rho_{p} d_{p} \phi \sqrt{T / \pi} f(\phi, e),
$$

where $\eta_{1}$ characterizes the stress-strain relation in the intermediate granular flow regime, in which particles often undergo long-lasting contacts, and $\eta_{2}$ accounts for the collisional contribution. The second contribute is insignificant in the dense flow regime, whereas the first term contribution becomes very small comparing to the second term as the solid fraction decreases below $0.9 \phi_{c}$. The comparison of the newly formulated constitutive law and DEM is given in Fig. 8, A satisfactory agreement with DEM is achieved by the constitutive law proposed in this paper.

[ Fig. 8 insert here. ]

The proposed correlation, formulated as a function of solid fraction and granular temperature, is able to account for the stress-strain relation in a wide range of inertial number from 0.003 to 0.2 in current research. The lower limit is close to the quasi-static regime, in which particle interacts with each other in the nature of long-lasting contacts. The viscosity 
diverges in $\left(1-\phi / \phi_{c}\right)^{-1.367}$ when the solid fraction $\phi$ approaches the maximum solid fraction as the inertial number decreases towards 0 . On the other side, the upper limit is at the edge of the transition from the intermediate regime to the collisional regime and a combination of particle enduring and collisional interactions coexists. The relative contribution of the two terms in Eq. (15) reflects the particle-scale interaction transition as the inertial number increases. A qualitative assessment of the generality of the scaling law in other flow configurations was provided in Appendix D.

It is also encouraging to notice that the constitutive law, which is derived from DEM observations, is very similar to the constitutive expression for the shear viscosity derived in a theoretical study by Savage [10]: the first term $\eta_{1} \sim d_{p} P / \sqrt{T}$ times a coefficient accounting for the divergence near the random close packing and a second term $\eta_{2} \sim \rho_{p} d_{p} \phi \sqrt{T} f(\phi, e)$. The similarities indicate that the theory developed by Savage correctly generates the constitutive law for the shear viscosity in the intermediate flow regime.

\section{Kinetic theory for dense granular flows}

The general governing equations for granular flows are written as

$$
\begin{aligned}
& \frac{\partial \phi}{\partial t}+\nabla \cdot(\phi \boldsymbol{v})=0 \\
& \rho_{p} \phi\left(\frac{\partial \boldsymbol{v}}{\partial t}+\boldsymbol{v} \cdot \nabla \boldsymbol{v}\right)=\nabla \cdot \boldsymbol{\sigma}+\rho_{p} \phi \boldsymbol{g} \\
& \frac{3}{2} \rho_{p} \phi\left(\frac{\partial T}{\partial t}+\boldsymbol{v} \cdot \nabla T\right)=-\nabla \cdot \boldsymbol{q}+\boldsymbol{\sigma}: \nabla \boldsymbol{v}-\chi
\end{aligned}
$$

where : is the contraction operator of two tensors, $\boldsymbol{q}$ is the heat flux, and $\chi$ represents the energy dissipation. Eqs. (9) and (10) are obtained by simplifying Eq. (17) as discussed in Section 3. The stress tensor $\boldsymbol{\sigma}$ is related to the strain tensor $\boldsymbol{S}$ as

$$
\boldsymbol{\sigma}=-P \boldsymbol{I}+2 \eta \boldsymbol{S}+\left(\eta_{b}-\frac{2}{3} \eta\right)(\nabla \cdot \boldsymbol{v}) \boldsymbol{I},
$$


with $\eta$ and $\eta_{b}$ are the shear viscosity and the bulk viscosity, separately. The heat flux is often written following Fourier's law:

$$
\boldsymbol{q}=-k \nabla T
$$

where $k$ is the thermal conductivity of granular kinetic energy.

Applying Eq. (18) to steady granular flows in the annular Couette cell leads to a simplified granular kinetic energy equation written as

$$
\tau \dot{\gamma}+k \nabla^{2} T-\chi=0
$$

So far, we have obtained the shear viscosity as shown in Eq. 15 from DEM simulations. Since we have verified that Savage's theoretical development leads to a similar form of the shear viscosity as revealed in our DEM simulations, the determinations of the heat flux $\boldsymbol{q}$ and $\chi$ are following Savage's research [10]. The thermal conductivity is determined from

$$
\frac{k}{\eta}=\frac{2\left[1+\frac{1}{4} \pi\left(G^{-2}+3 G^{-1}+9 / 4\right)\right]}{1+\frac{1}{8} \pi\left(G^{-2}+2 G^{-1}+1\right)},
$$

where $G=\phi g_{0}$. The thermal conductivity has been found to be well described up to high solid fraction by the kinetic theory [54]. Therefore, the calibration of $k$ as shown in Eq. (22) is used in this research.

The energy dissipation term is written as

$$
\chi=\frac{D \beta^{2}}{A d_{p}^{2}} \eta T,
$$

where, $A, D$ and $\beta$ are constants derived in Savage's theory. A discussion of the energy dissipation term and the parameters is provided later in this section.

The Savage's theory is based on the critical state solid mechanics, predicting that the ratio of the shear stress to the confining pressure is a constant in homogenous simple shear flows. To overcome this limitation, the equation of state is introduced to calculate granular 
pressure independently, which is formulated as [55, 15]

$$
P=\rho_{p} H(\phi, e) T
$$

where $H(\phi, e)$ is a function of solid fraction $\phi$ and restitution coefficient $e$, having the following formulation

$$
H(\phi, e)=\phi\left[1+2(1+e) \phi g_{0}\right]
$$

Chialvo and Sundaresan [15] proposed a correction of the radial distribution function $g_{0}$ in the dense regime, written as

$$
g_{0}^{C S}=\frac{1-\phi / 2}{\left(1-\phi^{3}\right)}+\frac{\alpha_{1} \phi^{2}}{\left(\phi_{c}-\phi\right)^{3 / 2}} .
$$

The assessment of the kinetic theory to capture nonlocal effects in dense granular flows is presented in the following.

\section{1. $\mu(I)$ law from the kinetic theory}

Substituting Eq. (24) into Eq. (15), the shear viscosity is rewritten as

$$
\eta=\rho_{p} d_{p} J(\phi, e) T^{1 / 2}
$$

with

$$
J(\phi, e)=a H(\phi, e)\left(1-\phi / \phi_{c}\right)^{-b}+\frac{\phi f(\phi, e)}{\sqrt{\pi}} .
$$

The equivalent shear stress $\tau$ can be written as

$$
\tau=\rho_{p} d_{p} \dot{\gamma} J(\phi, e) \sqrt{T}
$$

An effective friction coefficient $\mu^{K T}$ can be derived from Eq. (29) and (24), which is

$$
\mu^{K T}=\frac{J(\phi, e)}{H(\phi, e)} \frac{d_{p} \dot{\gamma}}{\sqrt{T}}
$$


Substituting Eq. (28) into Eq. (30), we obtain

$$
\mu^{K T}=a\left(1-\phi / \phi_{c}\right)^{-b} \frac{\dot{\gamma} d_{p}}{\sqrt{T}}+\frac{\phi f(\phi, e)}{\sqrt{\pi H(\phi, e)}} I .
$$

If $\dot{\gamma} d_{p} / \sqrt{T}$ is proportional to $\left(\phi_{c}-\phi\right)^{b}$, the first term will be a constant, Eq. 31 turns into a similar form as proposed by da Cruz et al. [8], which is written as

$$
\mu_{d a \text { Cruz }}=\mu_{s}+\alpha_{2} I
$$

Fig. 6 reveals that $\dot{\gamma} d_{p} / \sqrt{T}=c\left(\phi_{c}-\phi\right)^{1.6}$ with a power a little bit larger than $b$ (fitted $b=1.367)$. Eq. (31) is rewritten as

$$
\mu^{K T}=\alpha_{3}\left(\phi_{c}-\phi\right)^{b_{2}}+\frac{\phi f(\phi, e)}{\sqrt{\pi H(\phi, e)}} I .
$$

This equation suggests that the effective friction coefficient does not only depend on the inertial number $I$ but also is a function of $\phi_{c}-\phi$ in the shear cell, in which strong shear stress gradients exist.

Predicted $\mu^{K T}$ from Eq. (33) are compared with DEM simulation results as shown in Fig. 9. In Eq. (33), the constitutive coefficient $H(\phi, e)$ is calculated from a refitted radial distribution function $g_{0}^{C S M}$ which is formulated as

$$
g_{0}^{C S M}=\frac{1-1 / 2 \phi}{(1-\phi)^{3}}+\frac{\alpha_{1} \phi^{2}}{\left(\phi_{c}-\phi\right)^{3}} .
$$

Parameter $b_{2}$ is equal to 0.233 , determined from the slopes in Fig. 6 and 7, and $\alpha_{3}$ is equal to 0.68 .

[ Fig. 9 insert here. ]

The $\mu-I$ relationship at the inner wall of the vane shear cell is correctly predicted by replacing the first term $\alpha_{3}\left(\phi_{c}-\phi\right)^{b_{2}}$ in Eq. (33) with $\mu_{s}$, recalling that the $\mu$ - $I$ relationship at the inner wall follows the $\mu(I)$ law, which reads in Eq. (1) . The correct prediction of the $\mu-I$ relationship in the annular shear cell evidences that the developed model is able to 
recover the nonlocal effects in the $\mu-I$ relation. The proposed shear viscosity correlation is directly derived from DEM results, which already incorporates the nonlocal effects on the granular temperature profile. To recover $\mu-I$ relation in continuum modeling, the granular temperature profiles need to be correctly predicted in the first place by the granular kinetic energy equation.

The new formulation of the effective friction coefficient $\mu^{K T}$ in Eq. $\sqrt{33}$ indicates that the effective friction coefficient prediction in case of $\mu<\mu_{s}$ as $I \rightarrow 0$ is able to be recovered by introducing the compressibility in the form of $\left(\phi_{c}-\phi\right)^{b_{2}}$. The formulation fixed the problem in the classic $\mu(I)$ laws expressed in Eq. (1) and Eq. (32) that particle motion is not possible in the region of $\mu<\mu_{s}$. Comparing to nonlocal rheology models in Ref. [17, 22, the derived formulation is much simpler and the prediction performance is still satisfactory. The formulation seems to work for more complex granular flows such as bin flows in Ref. [56], in which $\mu=0.647\left(\phi_{c}-\phi\right)^{0.1975}$ is obtained in the region of $\mu<\mu_{s}$ (corresponding to $I<0.03$ in the reference). The obtained relation in the bin when $I \rightarrow 0$ complies with the proposed $\mu^{K T}$ formulation in our research. In addition, the fitted parameters are close to what is reported in Fig. 9. In the region of $\mu>\mu_{s}$, both terms in Eq. (33) contributes to the calculation of the effective friction coefficient and Fig. 9 shows that the formulation satisfactorily predicts the $\mu-I$ relation in this region.

\subsection{Granular kinetic energy dissipation in the dense regime}

For simple shear flows in steady state, the granular kinetic energy equation is reduced to

$$
\tau \dot{\gamma}-\chi=0
$$

With Eqs. (13), (27) and (23), we obtain

$$
\frac{T}{\left(\dot{\gamma} d_{p}\right)^{2}}=\frac{A}{D \beta^{2}} .
$$

To recover the general form of the energy dissipation term, which is formulated in previous 
research [52, 39, 35] and written as

$$
\chi=\frac{\rho_{p}}{d_{p}} K(\phi, e) T^{3 / 2}
$$

with

$$
K(\phi, e)=\frac{12}{\sqrt{\pi}} \phi^{2} g_{0}\left(1-e^{2}\right)
$$

$D \beta^{2} / A$ should be formulated as

$$
\frac{A}{D \beta^{2}}=\frac{J(\theta, e)}{K(\theta, e)}
$$

With Eq. (36), DEM simulation of simple dense granular flows suggests [15]

$$
\frac{A}{D \beta^{2}}=\alpha_{4}\left(\phi_{c}-\phi\right)^{-1 / 2}
$$

Here we define a correlation length $L$ as

$$
L=\frac{A d_{p}}{D \beta^{2}}=\alpha_{4}\left(\phi_{c}-\phi\right)^{-1 / 2} d_{p}
$$

Then the energy dissipation term in the dense regime can be written as

$$
\chi=\frac{\rho_{p}}{L} J(\theta, e) T^{3 / 2} .
$$

Calibration of parameter $\alpha_{4}$ and $L$ can be achieved by combining Eq. (39) and Eq. (40). For simplicity, we calibrate the correlation length with DEM simulation results of simple shear flows [15]

$$
\frac{L^{D E M}}{d_{p}}=\left(\frac{\phi_{c}-0.45}{\phi_{c}-\phi}\right)^{1 / 2} .
$$

To remain the nonelastic collision effects on the energy dissipation rate, $\chi$ can be rewritten as

$$
\chi=\frac{\rho_{p}}{L^{D E M}} J(\theta, e)\left(1-e_{e f f}\right) T^{3 / 2},
$$


where $e_{e f f}=e-3 / 2 \mu_{p} \exp \left(-3 \mu_{p}\right)$ is proposed by Chialvo and Sundaresan [15].

The dependency of the correlation length on the solid fraction is predicted by Eq. (43) and compared with Berzi and Jenkins's model [57, which is shown in Fig. 10. It is observed that the simplified length correlation model is capable of correctly predicting the correlation length in dense granular flows.

[ Fig. 10 insert here. ]

\subsection{Diffusion of granular temperature}

The nonlocal effects are often accounted for by introducing a diffusion term (written in Laplacian form) in constitutive model developments [50]. The diffusion term in the kinetic theory is written as $k \nabla^{2} T$ with $k=D_{T} \eta$. In the dense regime where $\phi \in[0.49,0.636]$, calculation of Eq. (22) indicates that parameter $D_{T}$ is equal to 4 with little variation.

Scaling $r$ by the particle size $d_{p}$ and recalling $\dot{\gamma}=-r \frac{\partial}{\partial r}\left(v_{\theta}(r) / r\right)$, Eq. 21 turns into

$$
\left(\tilde{r} \frac{\partial}{\partial \tilde{r}}\left(\frac{1}{\tilde{r}} v_{\theta}\right)\right)^{2}+\frac{D_{T}}{\tilde{r}} \frac{\partial}{\partial \tilde{r}}\left(\tilde{r} \frac{\partial T}{\partial \tilde{r}}\right)-\frac{1}{\tilde{L}} T=0,
$$

where, $\tilde{r}=r / d_{p}$ and $\tilde{L}=L^{D E M} / d_{p}$. The equation is numerically solved with a given Dirichlet boundary at $r=R_{i}$ and a Neumann boundary $\partial T /\left.\partial r\right|_{r=R_{o}}=0$. We approximated the Dirichlet boundary using the adjacent layer value $T_{r=R_{i}+d p}$ extracted from the DEM simulation.

The predicted granular temperature from the kinetic theory is plotted again the DEM results in Fig. 11. The correlation length adopted in the kinetic model are found to have the best fit with $\tilde{L}=0.522$ (corresponding to $\beta=0.352$ following Savage's energy dissipation equation). The correlation length less than the particle size indicates that the interparticle friction results in energy loss increase in the dense regime. The fitted correlation length value qualitatively matches with the $T /\left(\dot{\gamma} d_{p}\right)^{2}$ scaling law reported by Chialvo and Sundaresan [15]. 


\section{Discussions and conclusion}

The granular flows in the vane shear cell is numerically investigated by using DEM approach. The revealed flow properties such as shear stress and velocity profiles are consistent with 2D simulation results [16] and experimental measurements [23] in classic annular Cougranular kinetic energy equation is shown to be capable of predicting granular temperature profiles in the shear cell.

The influences of the material properties such as friction coefficient and restitution co- 
efficient on the flow properties are not fully explored in current research. Their influences on the shear viscosity is expected to be accounted for by calibrating the model parameters $a$ and $b$ in future works.

\section{Acknowledgments}

This study is conducted in the framework of the "PowderReg" project, funded by the European programme Interreg VA GR within the priority axis 4 "Strengthen the competitiveness and the attractiveness of the Grande Région / Großregion". 
[1] L. Staron, P.-Y. Lagrée, S. Popinet, Continuum simulation of the discharge of the granular silo, The European Physical Journal E 37 (1) (2014) 5.

[2] Q. Luo, Q. Zheng, A. Yu, Quantitative comparison of hydrodynamic and elastoplastic approaches for modeling granular flow in silo, AIChE Journal 65 (5) (2019) e16533.

[3] S. Rau, C. Nied, S. Schmidt, D. Niedziela, J. Lindner, K. Sommer, Multi-phase simulation of pneumatic conveying applying a hydrodynamic hybrid model for the granular phase, Powder Technology 330 (2018) 339-348.

[4] N. Gaudel, S. K. De Richter, Effect of vibrations on granular material flows down an inclined plane using dem simulations, Powder Technology 346 (2019) 256-264.

[5] Q. Zheng, A. Yu, Modelling the granular flow in a rotating drum by the eulerian finite element method, Powder Technology 286 (2015) 361-370.

[6] P. Abrahamsson, S. Sasic, A. Rasmuson, On continuum modelling of dense inelastic granular flows of relevance for high shear granulation, Powder Technology 294 (2016) 323-329.

[7] G. MiDi, On dense granular flows, The European Physical Journal E 14 (4) (2004) 341-365.

[8] F. Da Cruz, S. Emam, M. Prochnow, J.-N. Roux, F. Chevoir, Rheophysics of dense granular materials: Discrete simulation of plane shear flows, Physical Review E 72 (2) (2005) 021309.

[9] S. Chialvo, J. Sun, S. Sundaresan, Bridging the rheology of granular flows in three regimes, Physical Review E 85 (2) (2012) 021305.

[10] S. Savage, Analyses of slow high-concentration flows of granular materials, Journal of Fluid Mechanics 377 (1998) 1-26.

[11] M. Depken, W. van Saarloos, M. van Hecke, Continuum approach to wide shear zones in quasistatic granular matter, Physical Review E 73 (3) (2006) 031302.

[12] A. Singh, V. Magnanimo, K. Saitoh, S. Luding, The role of gravity or pressure and contact stiffness in granular rheology, New Journal of Physics 17 (4) (2015) 043028.

[13] B. Utter, R. P. Behringer, Self-diffusion in dense granular shear flows, Physical Review E 69 (3) (2004) 031308.

[14] T. Barker, D. G. Schaeffer, P. Bohórquez, J. Gray, Well-posed and ill-posed behaviour of the $\mu(\mathrm{I})-$ rheology for granular flow, Journal of Fluid Mechanics 779 (2015) 794-818.

[15] S. Chialvo, S. Sundaresan, A modified kinetic theory for frictional granular flows in dense and dilute regimes, Physics of Fluids 25 (7) (2013) 070603.

[16] G. Koval, J.-N. Roux, A. Corfdir, F. Chevoir, Annular shear of cohesionless granular materials: From the inertial to quasistatic regime, Physical Review E 79 (2) (2009) 021306.

[17] K. Kamrin, G. Koval, Nonlocal constitutive relation for steady granular flow, Physical Review Letters 108 (17) (2012) 178301. 
[18] A. Fall, G. Ovarlez, D. Hautemayou, C. Mézière, J.-N. Roux, F. Chevoir, Dry granular flows: Rheological measurements of the $\mu(\mathrm{I})$-rheology, Journal of Rheology 59 (4) (2015) 1065-1080.

[19] P. Jop, Y. Forterre, O. Pouliquen, A constitutive law for dense granular flows, Nature 441 (7094) (2006) 727.

[20] A. Franci, M. Cremonesi, 3D regularized $\mu$ (I)-rheology for granular flows simulation, Journal of Computational Physics 378 (2019) 257-277.

[21] D. Schaeffer, T. Barker, D. Tsuji, P. Gremaud, M. Shearer, J. Gray, Constitutive relations for compressible granular flow in the inertial regime, Journal of Fluid Mechanics 874 (2019) 926-951.

[22] M. Bouzid, M. Trulsson, P. Claudin, E. Clément, B. Andreotti, Nonlocal rheology of granular flows across yield conditions, Physical Review Letters 111 (23) (2013) 238301.

[23] Z. Tang, T. A. Brzinski, M. Shearer, K. E. Daniels, Nonlocal rheology of dense granular flow in annular shear experiments, Soft Matter 14 (16) (2018) 3040-3048.

[24] T. Barker, J. Gray, Partial regularisation of the incompressible $\mu(\mathrm{I})$-rheology for granular flow, Journal of Fluid Mechanics 828 (2017) 5-32.

[25] Q. Zhang, K. Kamrin, Microscopic description of the granular fluidity field in nonlocal flow modeling, Physical Review Letters 118 (5) (2017) 058001.

[26] T. Barker, D. Schaeffer, M. Shearer, J. Gray, Well-posed continuum equations for granular flow with compressibility and $\mu$ (I)-rheology, Proceedings of the Royal Society A: Mathematical, Physical and Engineering Sciences 473 (2201) (2017) 20160846.

[27] J. S. Fannon, I. R. Moyles, A. C. Fowler, Application of the compressible $i$-dependent rheology to chute and shear flow instabilities, Journal of Fluid Mechanics 864 (2019) 1026-1057.

[28] O. Pouliquen, Velocity correlations in dense granular flows, Physical Review Letters 93 (24) (2004) 248001.

[29] O. Baran, D. Ertaş, T. C. Halsey, G. S. Grest, J. B. Lechman, Velocity correlations in dense gravitydriven granular chute flow, Physical Review E 74 (5) (2006) 051302.

[30] A. V. Orpe, A. Kudrolli, Velocity correlations in dense granular flows observed with internal imaging, Physical Review Letters 98 (23) (2007) 238001.

[31] L. Staron, Correlated motion in the bulk of dense granular flows, Physical Review E 77 (5) (2008) 051304.

[32] L. Staron, P.-Y. Lagrée, C. Josserand, D. Lhuillier, Flow and jamming of a two-dimensional granular bed: Toward a nonlocal rheology?, Physics of Fluids 22 (11) (2010) 113303.

[33] O. Pouliquen, Y. Forterre, A non-local rheology for dense granular flows, Philosophical Transactions of the Royal Society A: Mathematical, Physical and Engineering Sciences 367 (1909) (2009) 5091-5107.

[34] I. S. Aranson, L. S. Tsimring, Continuum description of avalanches in granular media, Physical Review 
E 64 (2) (2001) 020301.

[48] E. Azéma, F. Radjai, Internal structure of inertial granular flows, Physical Review Letters 112 (7) (2014) 078001.

[49] R. C. Hurley, J. E. Andrade, Friction in inertial granular flows: competition between dilation and grain-scale dissipation rates, Granular Matter 17 (3) (2015) 287-295.

[50] M. Bouzid, A. Izzet, M. Trulsson, E. Clément, P. Claudin, B. Andreotti, Non-local rheology in dense granular flows, The European Physical Journal E 38 (11) (2015) 125.

[51] W. Losert, L. Bocquet, T. Lubensky, J. P. Gollub, Particle dynamics in sheared granular matter, 
Physical Review Letters 85 (7) (2000) 1428.

[52] D. Gidaspow, Multiphase flow and fluidization: continuum and kinetic theory descriptions, Academic press, 1994.

[53] D. Ma, G. Ahmadi, An equation of state for dense rigid sphere gases, The Journal of Chemical Physics 84 (6) (1986) 3449-3450.

[54] L. Bocquet, W. Losert, D. Schalk, T. Lubensky, J. Gollub, Granular shear flow dynamics and forces: Experiment and continuum theory, Physical review E 65 (1) (2001) 011307.

[55] V. Garzó, J. Dufty, Dense fluid transport for inelastic hard spheres, Physical Review E 59 (5) (1999) 5895.

[56] A. Bhateja, D. V. Khakhar, Analysis of granular rheology in a quasi-two-dimensional slow flow by means of discrete element method based simulations, Physics of Fluids 32 (1) (2020) 013301.

[57] D. Berzi, J. T. Jenkins, Fluidity, anisotropy, and velocity correlations in frictionless, collisional grain flows, Physical Review Fluids 3 (9) (2018) 094303.

[58] T. Weinhart, R. Hartkamp, A. R. Thornton, S. Luding, Coarse-grained local and objective continuum description of three-dimensional granular flows down an inclined surface, Physics of Fluids 25 (7) (2013) 070605 . 


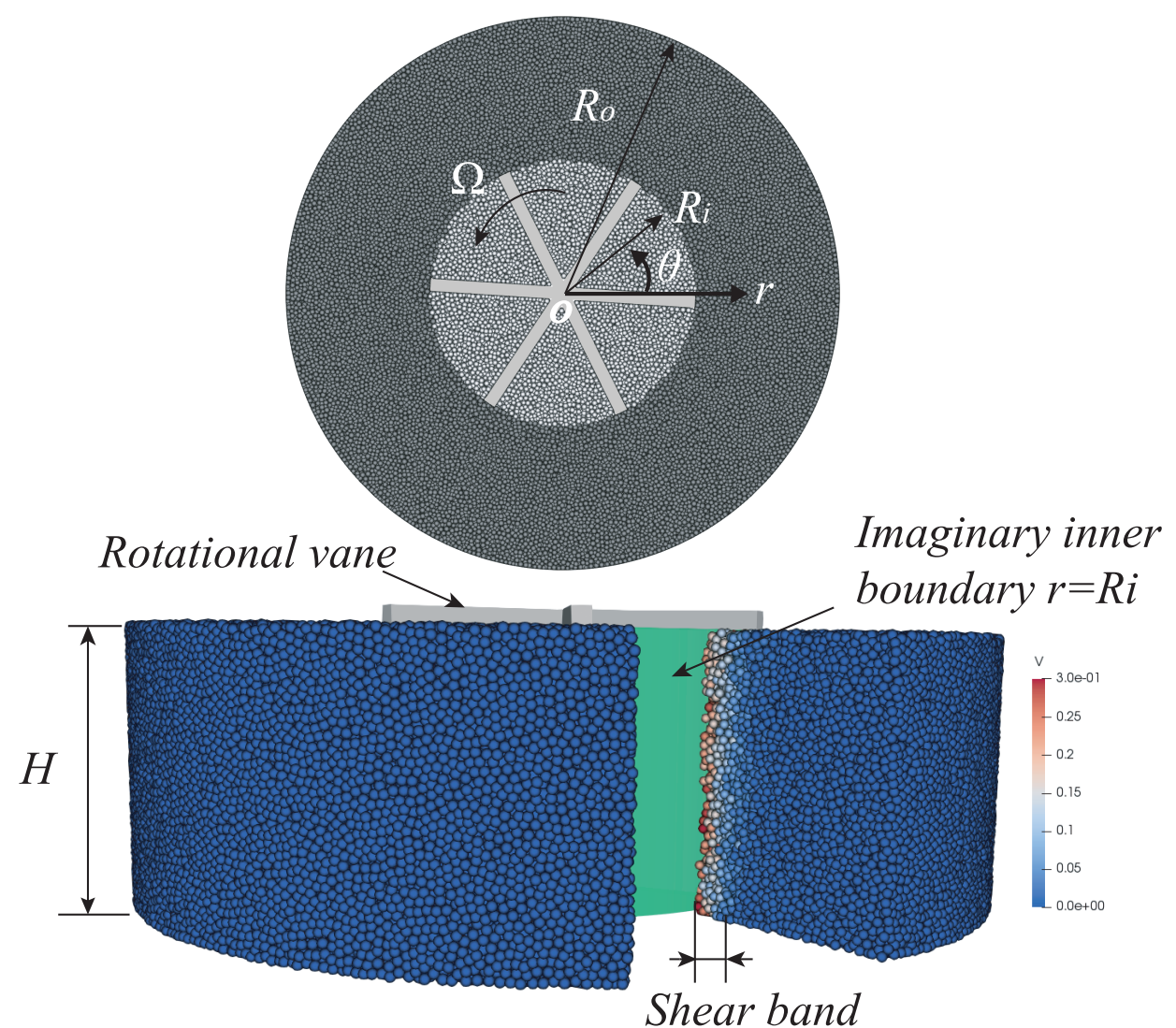

Figure 1: Geometry of the simulated system. 


\section{a)}
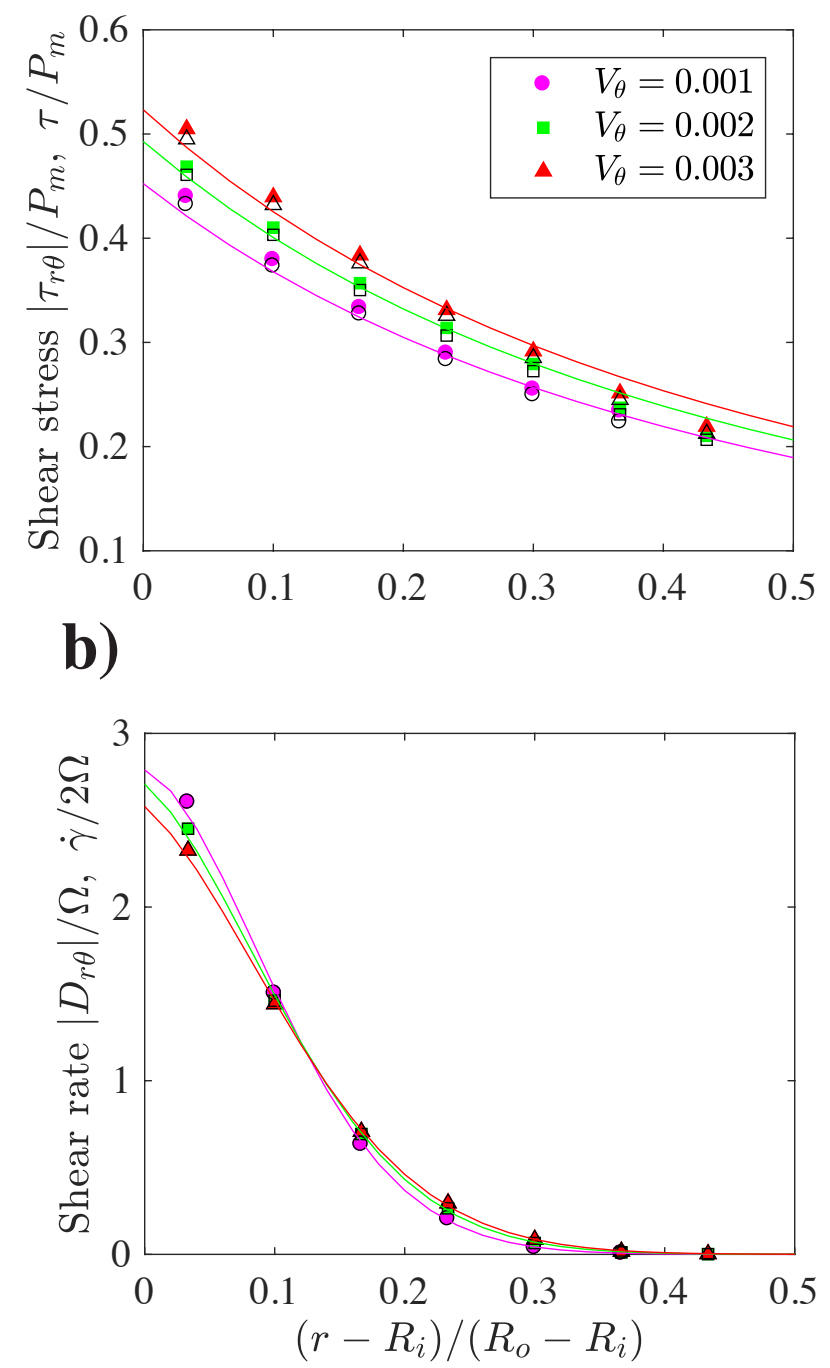

Figure 2: Dominance of $\tau_{r \theta}$ and $D_{r \theta}$ components in the calculation of the equivalent shear stress $\tau$ and the equivalent shear rate $\dot{\gamma}$. Filled symbols are equivalent shear stress and strain, whereas the open symbols (black) represent $\tau_{r \theta}$ and $D_{r \theta}$ components of $\boldsymbol{\tau}$ and $\boldsymbol{D}$ tensors. Three lines in (a) are fitted with formula $\tau / P_{m}=A / r^{2}$ with A equal to $0.4524 R_{i}^{2}, 0.4928 R_{i}^{2}$ and $0.5232 R_{i}^{2}$ from bottom to top. The lines in (b) are derived from fitted velocity equations. $P_{m}=0.6 \rho g H_{m}$ is the hydrostatic pressure at sampling depth $H_{m}=25 d_{p}$. 

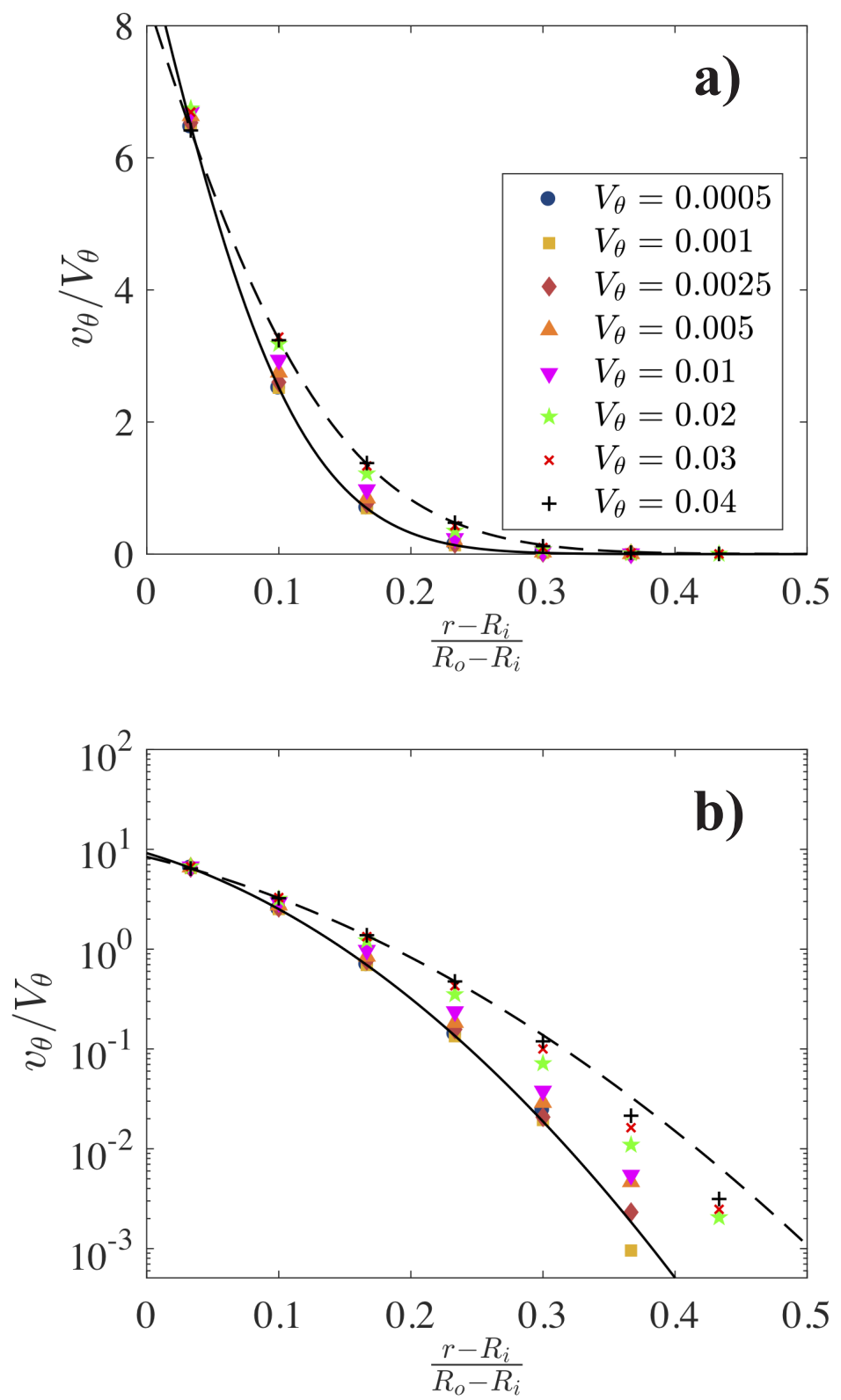

Figure 3: Velocity profiles in the shear cell at depth of $H_{m}=25 d_{p}$. The fitting of the dash line: $v_{\theta} / V_{\theta}=8.407 \exp \left(-7.406 \tilde{r}-21.02 \tilde{r}^{2}\right)$ with $\tilde{r}=\left(r-R_{i}\right) / R_{o}-R_{i}$. The fitting of the solid line: $v_{\theta} / V_{\theta}=9.1614 \exp \left(-9.054 \tilde{r}-38.6 \tilde{r}^{2}\right)$. 

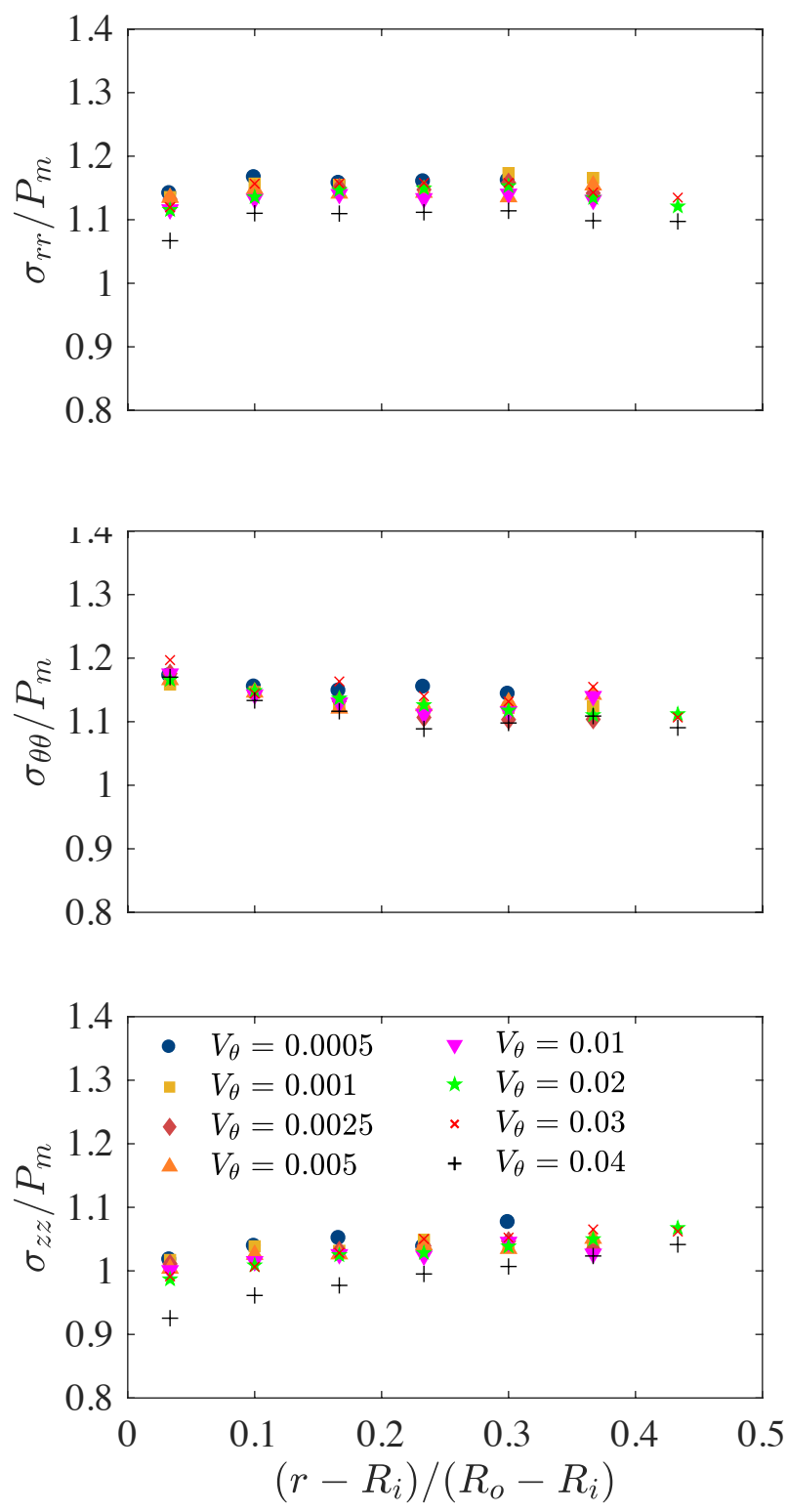

Figure 4: Profiles of normal stresses at depth of $H_{m}=25 d_{p} . P_{m}=0.6 \rho g H_{m}$ is the hydrostatic pressure. 

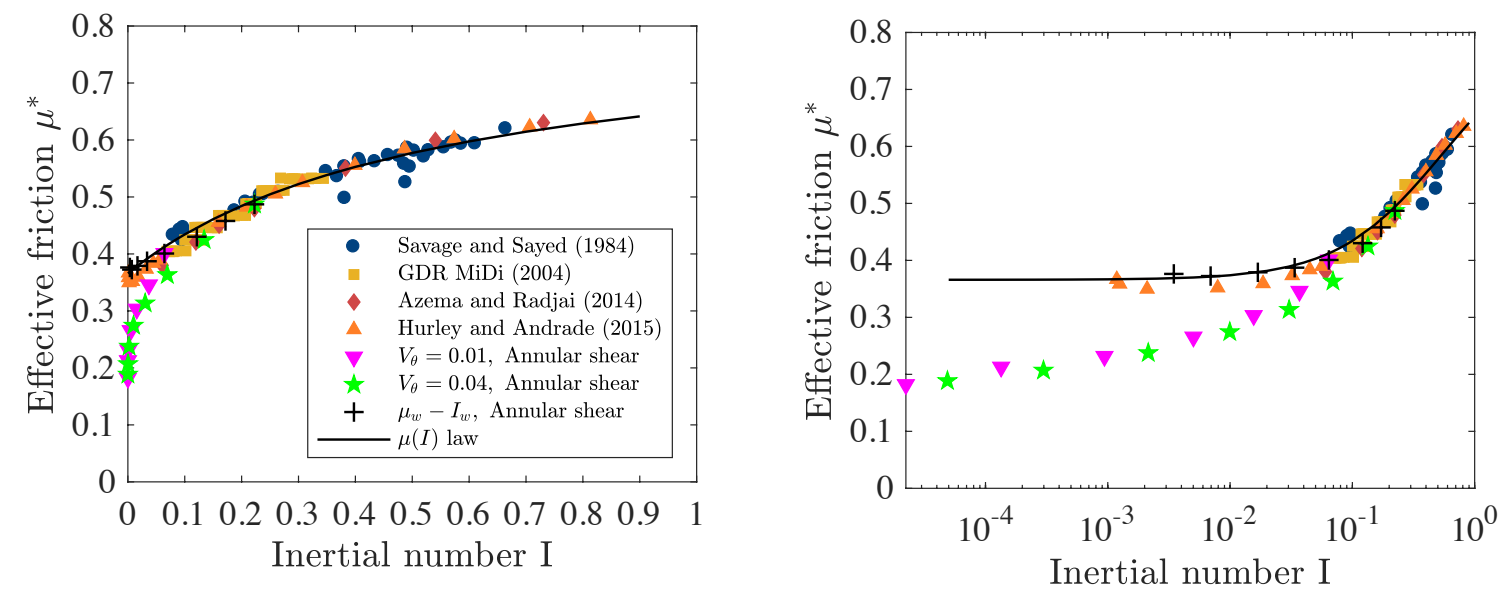

Figure 5: Relation between the effective friction coefficient and the inertial number. Flow configurations in the literature are: Savage and Syed (1984): Experimental measurements in an annular shear cell [47]; MiDi (2004): Experimental measurements on an inclined plane [7; Azema et al. (2014) and Hurley et al. (2015): 3D simulation of granular flows between two sheared planes [48, 49. $\mu_{w}$ and $I_{w}$ are measured at the inner boundary. Fitted parameters in Eq. (1) are: $\mu_{s}=\tan \left(20.1^{\circ}\right), \mu_{g}-\mu_{s}=0.4438$, and $I_{o}=0.55$.

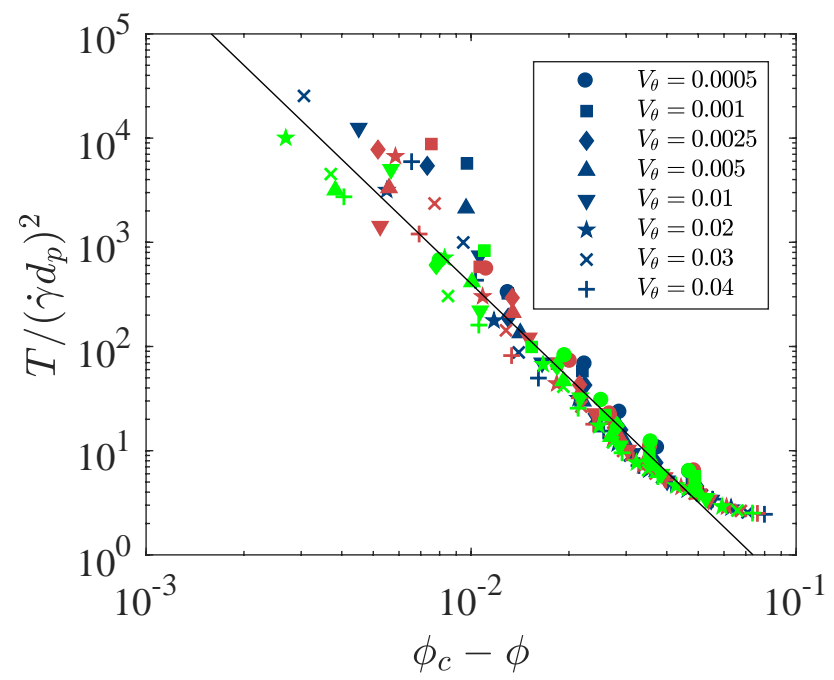

Figure 6: Relation of nondimensional granular temperature and solid fraction. Data is gathered at three depth levels: $H=20 d_{p}$ (Blue symbols), $H=25 d_{p}$ (Red symbols) and $H=30 d_{p}$ (Green symbols). Line: $T /\left(\dot{\gamma} d_{p}\right)^{2}=1 \times 10^{-4}\left(\phi_{c}-\phi\right)^{-3.2}$. 


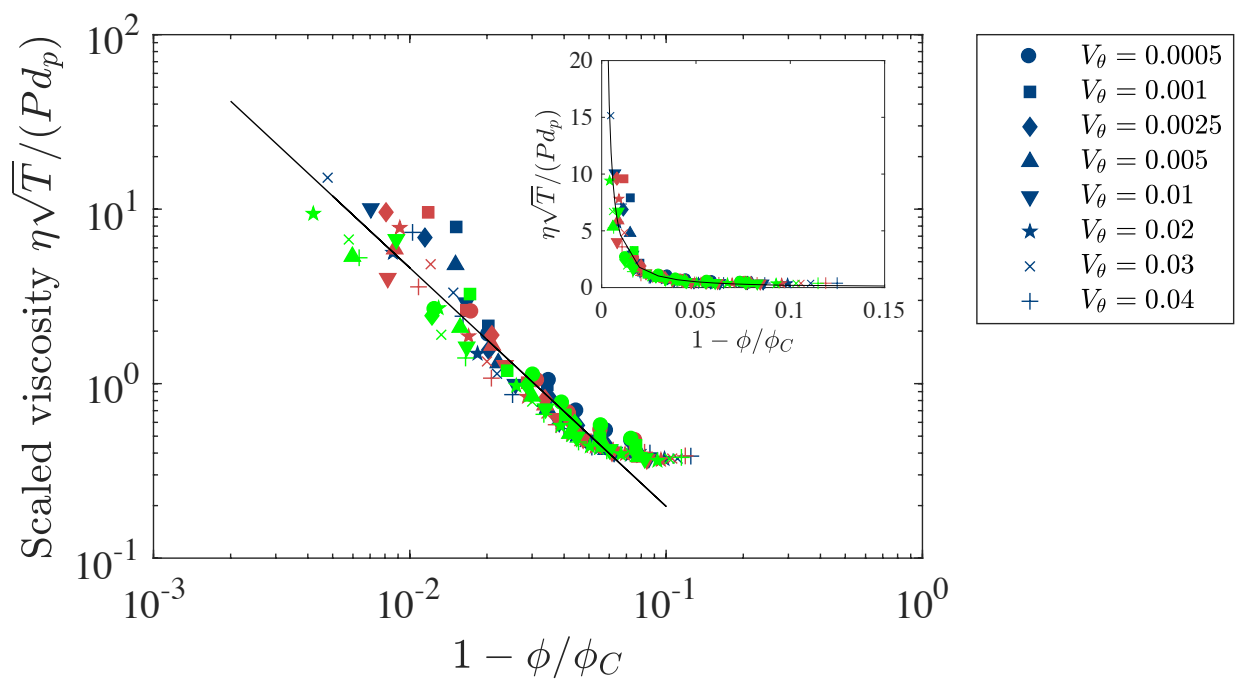

Figure 7: Scaling of dimensionless viscosity vs solid fraction variable $\left(1-\phi / \phi_{c}\right)$. Data is gathered at three depth levels: $H=20 d_{p}$ (Blue symbols), $H=25 d_{p}$ (Red symbols) and $H=30 d_{p}$ (Green symbols). $\phi_{C}$ is the critical solid fraction with the value of 0.64 (close random packing) in this research. The solid line: $\eta \sqrt{T} /(P d)=a\left(1-\phi / \phi_{c}\right)^{-b}$ with the estimated parameters $a=0.0085$ and $b=1.367$. 

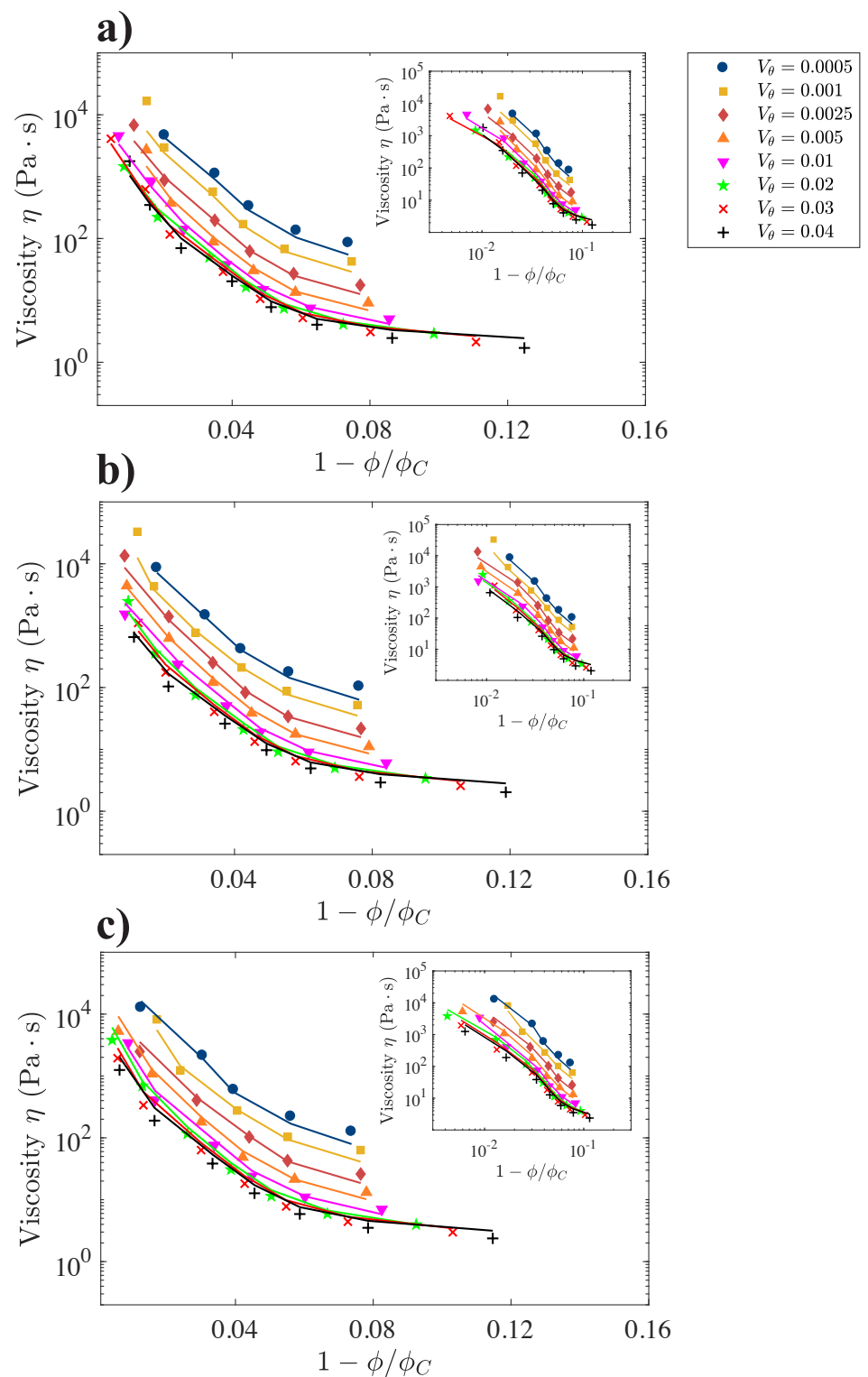

Figure 8: Viscosity from the constitutive law (solid lines) vs viscosity from DEM (symbols). Comparison is plotted at depth (a) $H=20 d_{p}$, (b) $H=25 d_{p}$ and (c) $H=30 d_{p}$. 


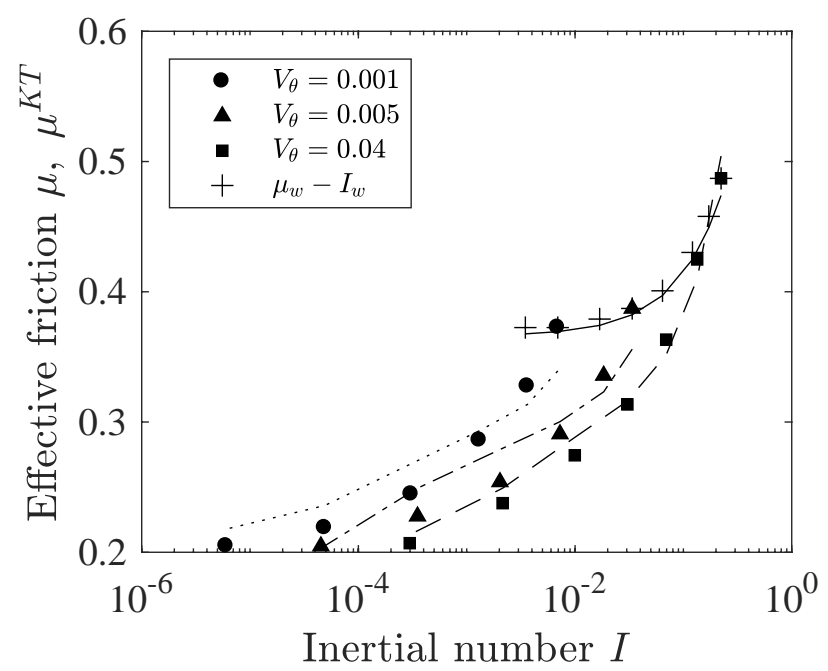

Figure 9: Comparison of predicted effective friction coefficient profiles with DEM simulation results. Markers represent $\mu-I$ relations revealed in DEM simulations of vane shear cell. Dash lines illustrate predictions from the proposed $\mu^{K T}$ equation (Eq. 33 in the text) with $\alpha_{3}=0.68$ and $b_{2}=0.233$. The solid line is predicted with $\mu^{K T}=\mu_{s}+\frac{\phi f(\phi, e)}{\sqrt{\pi H(\phi, e)}} I$.

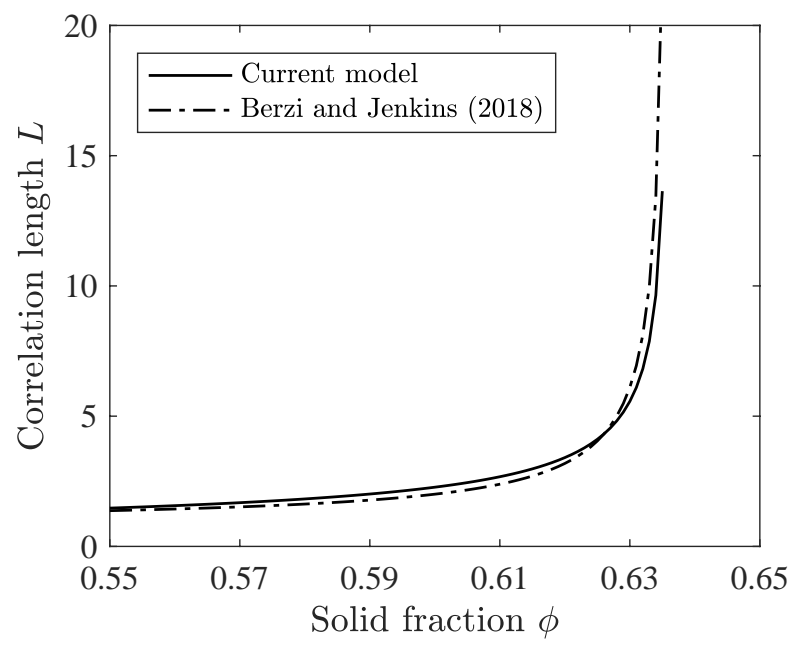

Figure 10: Comparison of correlation length predictions by current model and Berzi and Jenkins (2018). 


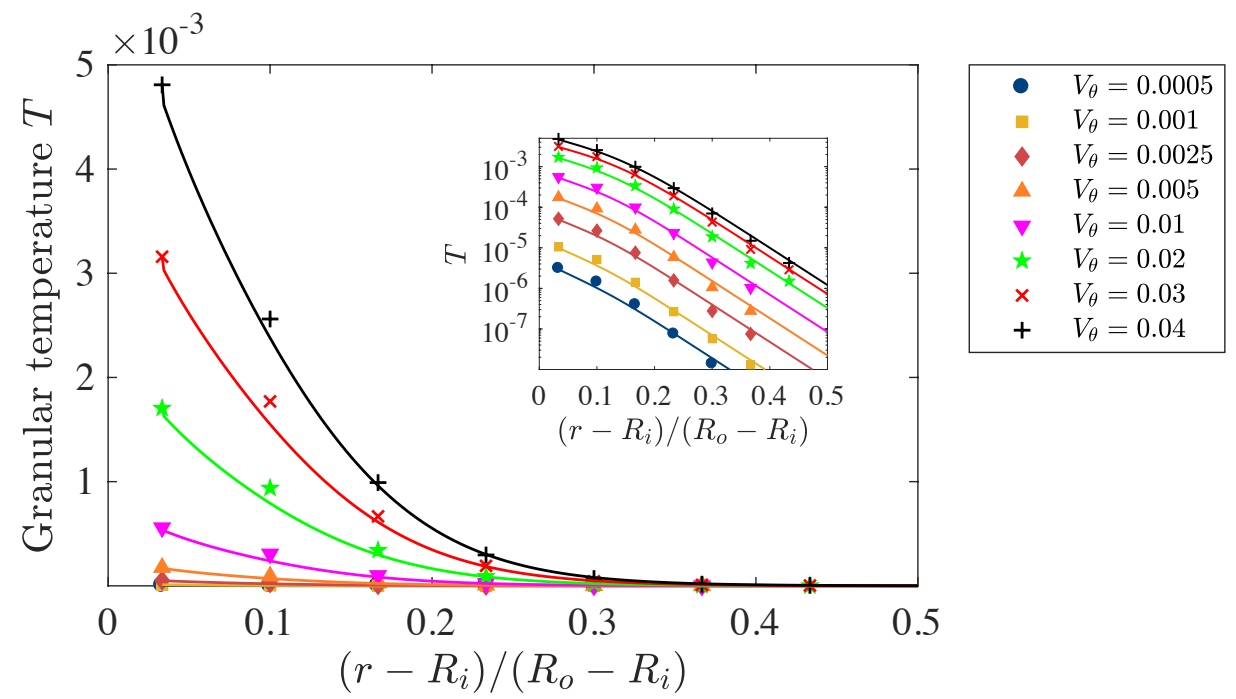

Figure 11: Granular temperature profiles in the shear cell. The inset plots the same granular temperature profiles in semi-log scale. Symbols are extracted at depth $H=25 d_{p}$ from the DEM simulation and the solid lines are predicted with the kinetic theory of dense granular flows. Parameters in the kinetic theory model: $D_{T}=4$ and $\tilde{L}=0.522$. 


\section{Appendix}

\section{A. Dilation of granular bed}

The solid volume fraction is an essential variable for describing dense granular flows. The bed height is a good indicator of the bulk solid fraction. The variation of the granular bed height with the dimensionless shear velocity in the annular cell is shown in Fig. A.1. The bed rise is not significant when the dimensionless shear velocity is less than 0.04 , above which the dilation of the bed starts to become noticeable, resembling the fluidization process in a fluidized bed. In this research, since the flow characteristics in the intermediate regime is considered, we limited the dimensionless shear velocity up to $V_{\theta}=0.04$.
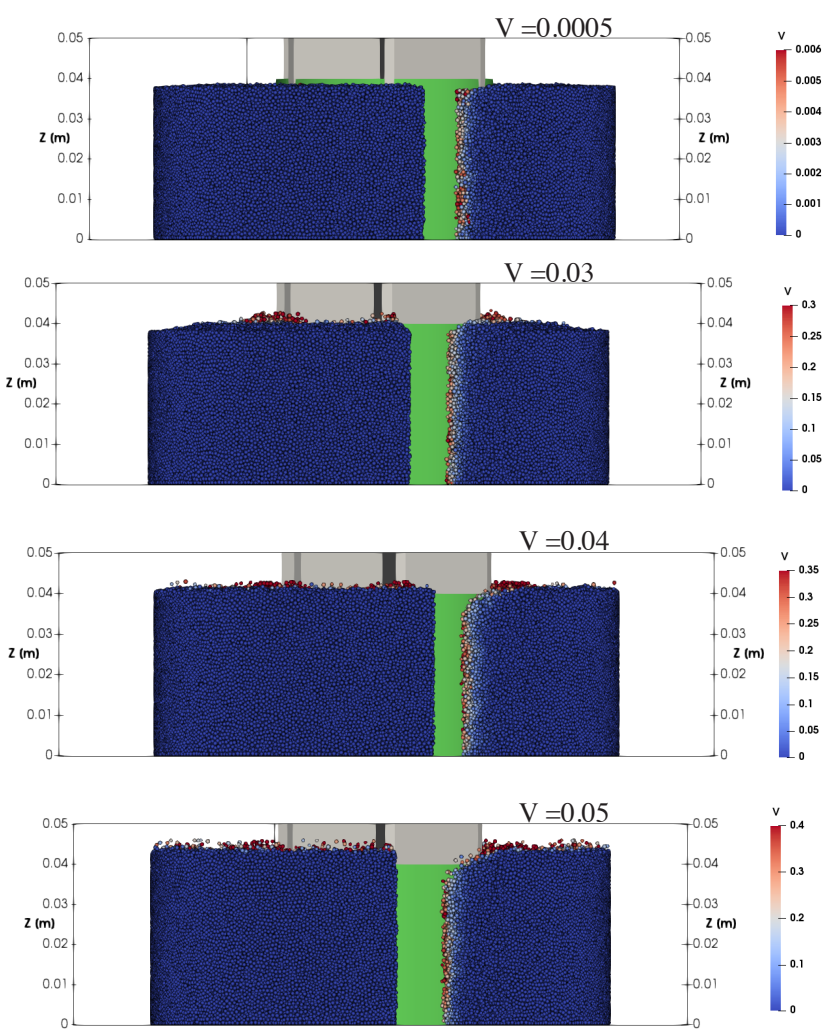

Figure A.1: Dilation of the granular bed in the vane shear cell. 


\section{B. Determination of particle bed rigidity}

A stiffness number characterizing the compressibility of granular material is defined following Singh et al. [12, which is written as

$$
\kappa=\sqrt{\frac{P d_{p}}{k_{n}}}
$$

where, $k_{n}$ is normal stiffness parameter in the contact model and $P$ is confining pressure. For Hertz-Mindlin contact model, the dimensionless stiffness number is derived as

$$
\kappa=C\left(P_{m} / Y\right)^{1 / 3},
$$

where, $P_{m}$ is a characteristic pressure calculated from $P_{m}=0.6 \rho_{p} g H_{m}$ in the modeled shear cell, $C$ is a constant. The derivation of constant $C$ is carried out by assuming that a pair of identical particles undergo a constant pressure $P_{m}$ acting on the cross section at the equator. The overlap $\delta$ between the two particles can be solved with the following two equations:

$$
\begin{aligned}
& F_{n}=P_{m} \pi R^{2}, \\
& F_{n}=\frac{4}{3} \times \frac{Y}{2\left(1-\nu^{2}\right)} \times \sqrt{\frac{R}{2}} \delta^{3 / 2},
\end{aligned}
$$

where $R$ is the radius of sphere particles. By defining $k_{n}=\frac{4}{3} \times \frac{Y}{2\left(1-\nu^{2}\right)} \sqrt{\delta}, k_{n}$ is solved as

$$
k_{n}=\frac{\sqrt{2}}{3} \frac{1}{\left(1-\nu^{2}\right)^{2 / 3}} \times\left[\frac{2 \sqrt{2}}{3} \pi\right]^{1 / 3}\left(P_{m} Y^{2}\right)^{1 / 3} R .
$$

Substituting $k_{n}$ into Eq. (B.1), we could obtain Eq. (B.2), in which $C$ is determined by material Poisson coefficient. For the glass material, the constant is estimated to be 1.69.

The results at two dimensionless stiffness numbers $\kappa=0.044$ and $\kappa=0.02$, which correspond to Young's modulus of $2 \times 10^{7}$ and $2 \times 10^{8}$ separately, are compared in Fig. B.1. The result differences are negligible in terms of equivalent shear stress and strain, which indicates that the adoption of $2 \times 10^{7}$ for Young's modulus is reasonable, considering both result accuracy and computational cost. 


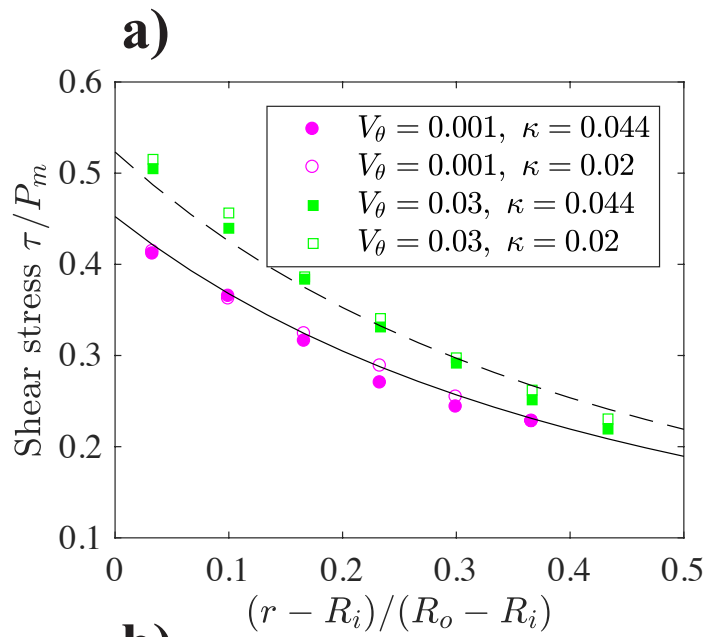

b)

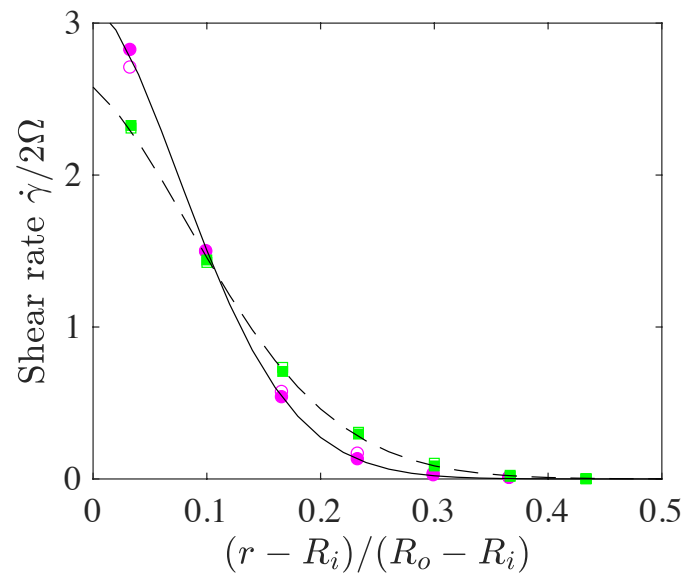

Figure B.1: Effects of the stiffness number on the equivalent shear stress and shear rate. Two lines in (a) are fitted with formula $\tau / P_{m}=A / r^{2}$ with A equal to $0.4524 R_{i}^{2}$ for the solid line and $0.5232 R_{i}^{2}$ for the dash line. The lines in (b) are derived from fitted velocity formulas. $P_{m}=0.6 \rho g H_{m}$ is the hydrostatic pressure at sampling depth $H_{m}=25 d_{p}$.

\section{Spatial size interval for sampling}

We used a classical averaging approach as used in many studies to derive kinematic properties from DEM simulation of granular flows [8, 16, 12, 46]. The determination of the averaging spatial size needs to ensure that the extracted kinematic property fields are smooth, which is called the local homogeneity assumption in Ref. [46]. Weinhart et al. [58] looked into the averaging length scale influences on the macroscopic fields and they concluded that the length scale $w \approx d_{p}$ leads to smooth macroscopic fields. The influence of 
the spatial interval on the solid fraction and velocity profiles in the shear cell is investigated and the comparison of the results obtained at two spatial size is shown in Fig. C.1. While the results of solid fraction and velocity remains relatively the same between $\Delta=1.2 d_{p}$ and $\Delta=2.0 d_{p}$, the solid fraction seems to fluctuate a little bit more at $\Delta=1.2 d_{p}$ at low shear velocity. For smoothness, $\Delta=2.0 d_{p}$ is adopted in this research.

The adopted spatial interval size of $2 d_{p}$ is also equal to the averaging spatial sizes reported in previous research [16, 12, 46]. Moreover, the selection of the sampling interval was validated by the relation matching of derived variables. For example, in the annular Couette cell, the equivalent shear rate is related to the azimuthal velocity as $\dot{\gamma}=-r \frac{\partial}{\partial r}\left(v_{\theta}(r) / r\right)$. It is clearly observed in Fig. 2 (b) that the profiles of the equivalent shear rate obtained from Eq. (8) match with the shear rate calculated from the azimuthal velocity correlations derived from DEM data. Therefore, we conclude that the time-volume averaging method with the spatial interval size of $2 d_{p}$ complies with the local homogeneity assumption.

\section{Generality of the $\eta \sqrt{T} /\left(P d_{p}\right) \sim\left(1-\phi / \phi_{c}\right)$ scaling relation}

Fig. D.1 shows a comparison of the obtained results from DEM simulations of the vane shear cell (markers and the dash line) and three flow configurations (the red solid line) including planar shear, planar shear with gravity and chute flows reported in Ref. [25]. The red solid line presents the well fitted model in Ref. 25 from the DEM simulations of dense granular flows in the three flow configurations mentioned above, realizing that $\eta \sqrt{T} /\left(P d_{p}\right)=\sqrt{T} /\left(g d_{p}\right)$ with $g$ being the fluidity parameter. A similar relation trend is observed between the scaled viscosity $\eta \sqrt{T} /\left(P d_{p}\right)$ and $1-\phi / \phi_{C}$ as the relation reported in our vane shear cell. The differences in the slope when $\phi \rightarrow \phi_{c}$ is believed to result from the influences of the friction coefficient $\mu_{D E M}$ and restitution coefficient $e$ parameters. The result indicates that Eq. (15) serves as a good correlation for the shear viscosity for dense granular flows and the influences of the material mechanical properties such as the friction coefficient and restitution coefficient needs to be taken into account by the parameters $a$ and $b$ in Eq. (15) in future work. 
a)
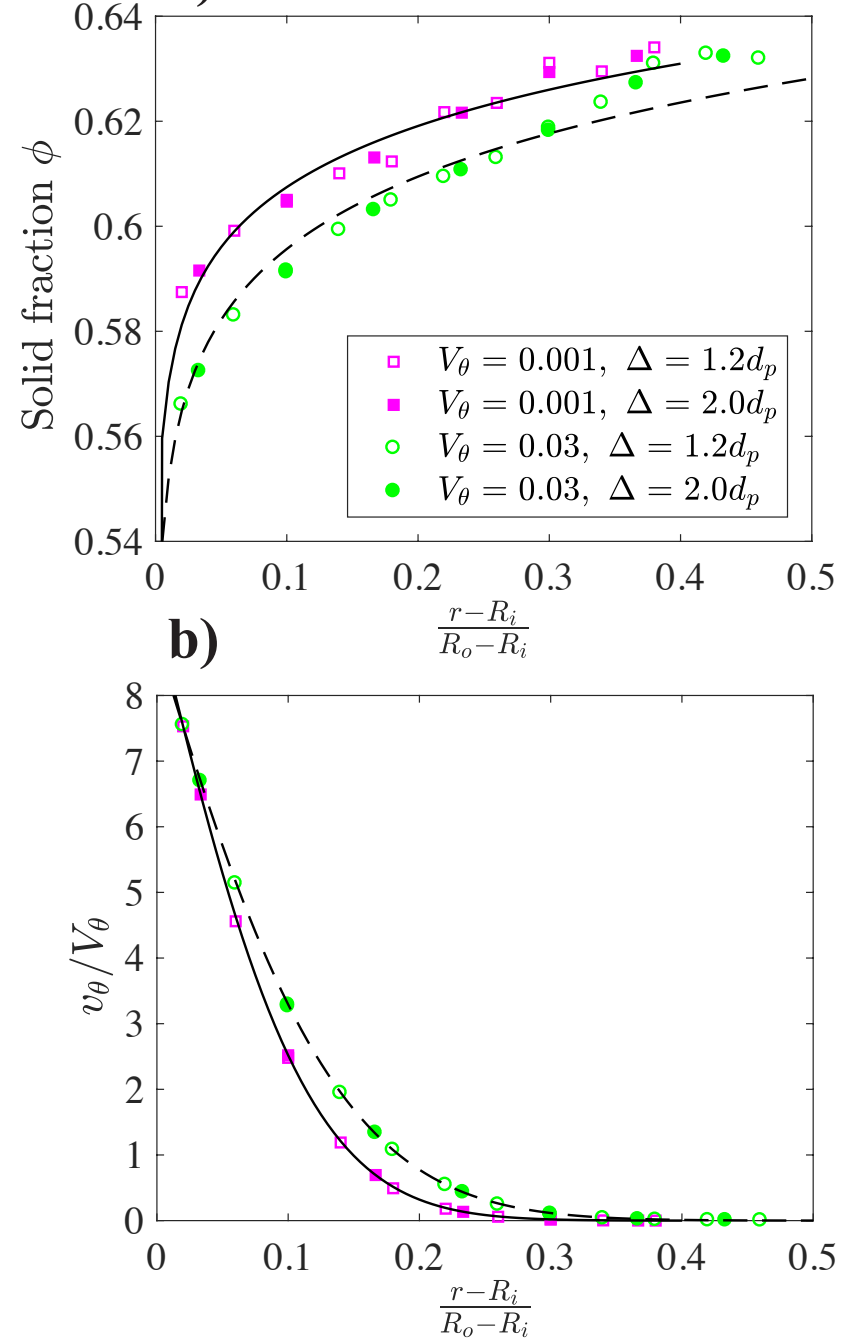

Figure C.1: Effects of the spatial sampling interval on the solid fraction and azimuthal velocity. Fitted lines in (a): $\phi=0.647 x^{0.02739}$ with $x=\left(r-R_{i}\right) /\left(R_{o}-R_{i}\right)$ for the solid line, $\phi=0.6427 x^{0.033}$ for the dash line. Fitted lines in (b): $v_{\theta} / V \theta=\exp \left(2.215-9.054 x-38.6 x^{2}\right)$ for the solid line, $v_{\theta} / V \theta=\exp \left(2.179-7.58 x-22.93 x^{2}\right)$ for the dash line. 


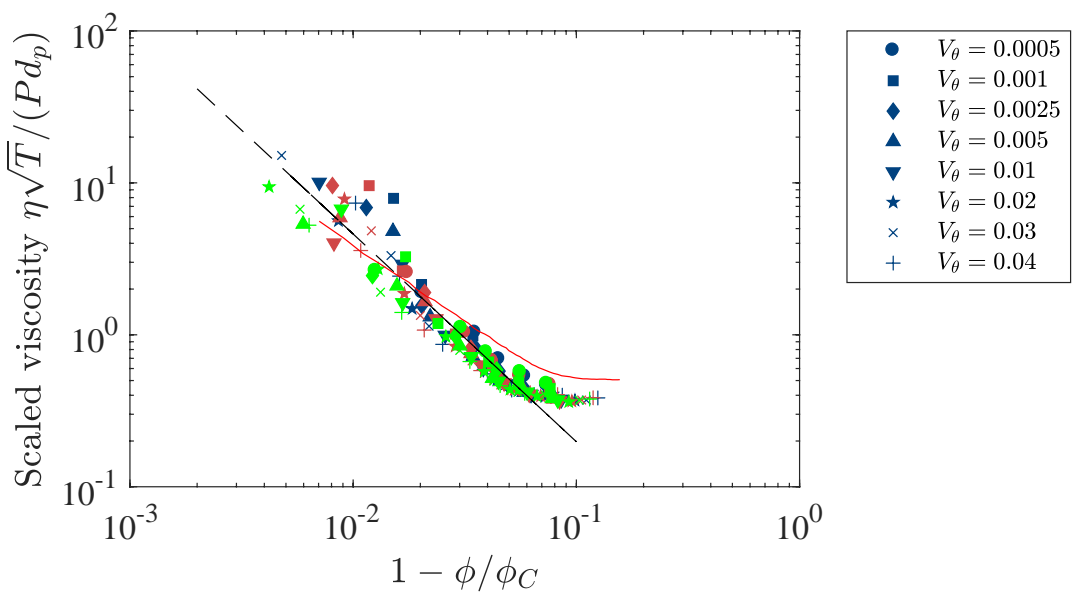

Figure D.1: Comparison of $\eta \sqrt{T} /\left(P d_{p}\right)$ V.S. $\left(1-\phi / \phi_{c}\right)$ scaling relation in various configurations. The markers present the obtained results in the vane shear cell at various rotational speeds and the dash line shows the fitted relation in our study. The solid red line shows the model reported in Ref. [25], which is fitted from DEM simulations of granular flows in three configurations: planar shear, planar shear with gravity and chute flows. $\phi_{c}$ is determined to be 0.63 in Ref. 25. 